\title{
Dévolution et politiques de santé en Écosse : un modèle de continuité et d'efficacité ?
}

Devolution and Health Policy in Scotland: A Model for Sustainability and

Efficiency?

\section{Edwige Camp-Pietrain}

\section{OpenEdition}

\section{Journals}

Édition électronique

URL : http://journals.openedition.org/rfcb/4258

DOI : $10.4000 /$ rfcb.4258

ISSN : 2429-4373

Éditeur

CRECIB - Centre de recherche et d'études en civilisation britannique

Référence électronique

Edwige Camp-Pietrain, « Dévolution et politiques de santé en Écosse : un modèle de continuité et d'efficacité ? », Revue Française de Civilisation Britannique [En ligne], XXIV-3 | 2019, mis en ligne le 30 août 2019, consulté le 04 septembre 2019. URL : http://journals.openedition.org/rfcb/4258 ; DOI : $10.4000 /$ rfcb.4258

Ce document a été généré automatiquement le 4 septembre 2019

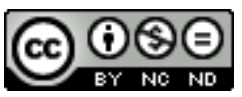

Revue française de civilisation britannique est mis à disposition selon les termes de la licence Creative Commons Attribution - Pas d'Utilisation Commerciale - Pas de Modification 4.0 International. 


\title{
Dévolution et politiques de santé en Écosse : un modèle de continuité et d'efficacité?
}

\author{
Devolution and Health Policy in Scotland: A Model for Sustainability and \\ Efficiency?
}

Edwige Camp-Pietrain

\section{Introduction}

1 Si le National Health Service (NHS) fut créé en 1948, certains de ses principes étaient déjà appliqués en Écosse ${ }^{1}$. Sa branche écossaise (Scottish Health Service) fut placée sous l'autorité du Secrétaire d'État à l'Écosse, qui pouvait réclamer des aménagements des politiques britanniques. La dévolution du pouvoir devait permettre sa gestion par un gouvernement et un Parlement écossais. L'espoir d'amélioration, présent dès les projets des années soixante-dix, s'est amplifié en réaction aux politiques néolibérales de Margaret Thatcher et de John Major ${ }^{2}$. Or, les besoins étaient immenses, l'Écosse étant décrite comme la «malade» de l'Europe occidentale ${ }^{3}$, en raison de taux de mortalité élevés. Cela résultait du mode de vie (alimentation, consommation d'alcool et de tabac, manque d'exercice physique), aggravé, pour les personnes modestes, par les conditions de vie (emploi, revenu). S'est ensuite ajouté un vieillissement de la population.

Depuis l'avènement de la dévolution, en 1999, la santé constitue la préoccupation centrale des dirigeants écossais. Les gouvernements SNP d'Alex Salmond (2007-2014) puis de Nicola Sturgeon ne se sont guère démarqués de leurs prédécesseurs, les coalitions travailliste/libéral-démocrate (dirigées par Jack McConnell à partir de 2001). Leurs choix politiques ont pu apparaître comme des modèles ${ }^{4}$. Même les Conservateurs, longtemps hostiles à la dévolution, ont dû s'adapter au consensus fréquent au Parlement de Holyrood. 
En 2018, lors des célébrations des 70 ans de NHS Scotland et des vingt ans du Scotland Act de 1998 portant création des institutions dévolues, cet article propose un état des lieux au regard des objectifs fixés par la Convention constitutionnelle qui conçut le projet de dévolution en 1995 : réformer l'organisation et le financement, instaurer des politiques distinctes, améliorer la santé5. Il s'agira de mettre en évidence, à partir de débats parlementaires, les spécificités écossaises dans un contexte assombri par les politiques britanniques d'austérité et par un Brexit rejeté par les Écossais lors du référendum de 2016.

\section{La dévolution, moyen de préservation d'un service public de santé ?}

4 Le NHS écossais, priorité budgétaire, est centré sur des structures publiques. La population porte néanmoins un regard critique sur sa gestion.

\section{Une priorité financière}

5 Le gouvernement SNP a consacré au NHS $43 \%$ de son budget en 2016-2017, soit 12,9 milliards de livres ${ }^{6}$. Sur dix ans, la progression a atteint $38 \%$, ou $8 \%$ en livres constantes. Les dépenses par habitant sont supérieures de près de $10 \%$ à celles de l'Angleterre. L'écart se retrouve sur le terrain, à travers le nombre de médecins généralistes pour mille habitants $(0,95$ contre 0,75$)$ ou le nombre de lits à l'hôpital' .

6 Cet avantage, dû à des facteurs sociaux (pauvreté relative) et géographiques (dispersion de la population), est aussi lié à une volonté politique. Dans les années soixante-dix, face à la percée du SNP indépendantiste, le gouvernement britannique conçut une formule (Barnett) garantissant la progression du budget écossais, indexée sur l'évolution des dépenses publiques pour l'Angleterre. La somme obtenue a été gérée en Écosse, d'abord par le Secrétaire d'État à l'Écosse, et, depuis 1999, par le gouvernement écossais, qui, libres de procéder à des arbitrages entre leurs domaines de compétences, ont privilégié la santé. Mais l'envolée des dépenses de santé sous Tony Blair a érodé l'avantage de l'Écosse (l'écart dans les dépenses par habitant atteignant auparavant $20 \%$ ). Ensuite, les politiques d'austérité de David Cameron ont entrainé une moindre progression. Ainsi, en 2017-2018, la hausse de $1,5 \%$ du budget écossais de santé correspond à une baisse de $0,1 \%$. Le gouvernement SNP dénonce les attaques de "Londres", tandis que ses adversaires conservateurs fustigent sa gestion, les Travaillistes déplorant les coupes infligées aux collectivités territoriales ${ }^{8}$. Ces tensions ne devraient pas fléchir car le Scotland Act de 2016 a doté les institutions écossaises de pouvoirs fiscaux substantiels qui imposeront des arbitrages entre impôts et dépenses.

\section{Les health boards au cœur du système}

7 Le gouvernement écossais alloue la quasi-totalité de ce budget aux 14 health boards régionaux, selon une formule qui tient compte du profil démographique et socioéconomique de leur bassin de population. Il veille à l'équilibre et sanctionne les manquements 9 . À la fin de 2018, face à la dégradation de leurs bilans, il a annoncé la prise en charge de leurs dettes, tout en assouplissant les exigences comptables ${ }^{10}$. 
Or, ces health boards sont au cœur du système depuis la loi de 1972 qui intégra les structures existantes ${ }^{11}$. Leur mise en concurrence sous les gouvernements conservateurs, peu appréciée, fut de courte durée ${ }^{12}$. Dès 1998, le gouvernement Blair restaura leur rôle en matière de stratégie, affaiblit les trusts, tandis que les médecins généralistes, peu désireux de gérer leurs budgets, furent incités à former des coopératives locales ${ }^{13}$. En 2004, la coalition de McConnell supprima les trusts et proscrivit les hôpitaux publics autonomes (foundation trusts), créés en Angleterre. Après avoir retrouvé une place centrale, les health boards ont dû se rapprocher des collectivités territoriales chargées de l'aide sociale, sous forme de community health partnerships et d'autorités « intégrées » en 2016. Celles-ci reçoivent $45 \%$ du budget des health boards pour financer les services délégués, notamment les « soins primaires » autour des médecins généralistes. Il s'agit de réduire les séjours à l'hôpital afin de libérer des lits (dans les 14 jours suivant l'autorisation médicale) ${ }^{14}$.

9 Le secteur privé suscite une défiance persistante. Après 1979, si les souscripteurs à des assurances privées ont augmenté, le nombre de lits dans le secteur privé commercial est resté circonscrit. L'ouverture à la concurrence des services annexes a fait l'objet d'une application tardive et partielle ${ }^{15}$.

10 S'agissant des soins, le recours par NHS Scotland aux sociétés privées reste l'exception, destinée à faire face à un besoin conjoncturel. Il ne peut être écarté en raison des pressions financières, les dépenses de personnel représentant $50 \%$ du budget des health boards. Certes, la ministre Shona Robison se prévalait en 2018 d'une hausse des effectifs depuis 2007 (12 400 sur un total de 160 000), facilitée par l'absence de frais universitaires pour les études médicales (à la différence de l'Angleterre) et la négociation de conditions de travail en Écosse (assurant un salaire minimum et des progressions supérieurs à

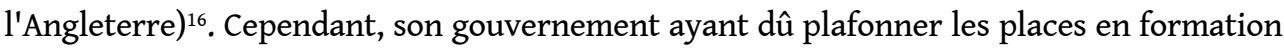
et les hausses de salaires, ce personnel restait insuffisant au regard des besoins. Les tensions se traduisent par un absentéisme croissant, des postes non pourvus, notamment en milieu rural (tableau 4). Cela oblige les health boards à recruter du personnel intérimaire coûteux. En radiologie, secteur le plus déficitaire, ils font appel à des sociétés privées installées à l'étranger. Le projet de loi publié en 2018, exigeant l'affectation du nombre requis de personnel qualifié, aura une influence sur la répartition, mais pas sur le recrutement ou la fidélisation.

11 Cet attachement aux structures publiques alimente une peur de la privatisation venue d'Angleterre, instrumentalisée lors des référendums de 2014 sur l'indépendance et de 2016 sur le maintien dans l'UE. Le SNP a dénoncé les politiques anglaises (recours à des sociétés privées, frais à la charge des patients) qui entraînent une réduction de la dotation budgétaire à l'Écosse, tout en s'inquiétant du risque d'ouverture à la concurrence de services publics dans le cadre d'accords de libre échange signés à l'issue du Brexit ${ }^{17}$.

12 Qui plus est, les organismes représentatifs du personnel médical ${ }^{18}$ craignent une aggravation des pénuries, les médecins et les infirmières originaires de l'UE représentant 5 à $10 \%$ des professionnels de santé en Écosse, $8 \%$ du secteur de l'aide à la personne. Certes, le gouvernement écossais dispose d'atouts, la gratuité des études supérieures étendue aux ressortissants de l'UE, la promesse de prise en charge des frais de dossier de régularisation des salariés du secteur public après le Brexit, et un discours enthousiaste quant à l'apport de ces personnes ${ }^{19}$. Ces facteurs ont permis au NHS écossais d'échapper à l'effondrement du nombre d'infirmiers arrivant de l'UE dans l'année suivant le 
référendum de 2016. Mais à l'issue du Brexit, l'Écosse sera soumise à la politique britannique d'immigration, appelée à se durcir alors que nombre de professions médicales écossaises, déjà reconnues comme souffrant de pénurie, sont autorisées à recruter dans des pays tiers (avec des quotas). Qui plus, même dans les domaines dévolus, le gouvernement britannique veut imposer un cadre commun, notamment pour la reconnaissance mutuelle des diplômes ou de la couverture sociale.

\section{Le regard critique des usagers} dix. Dans les années 2000, l'attente pour les opérations planifiées, qui pouvait excéder six mois, a été progressivement réduite, notamment grâce au rachat, en 2002, d'une clinique privée à Clydebank (devenue le Golden Jubilee Hospital) et à divers artifices comptables ${ }^{20}$. En 2011, Sturgeon a fait figurer ses objectifs dans une loi, approuvée à l'unanimité, garantissant notamment 12 semaines pour une chirurgie ${ }^{21}$. Depuis 2013, la situation ne cesse de se dégrader. En 2018, les délais d'attente ne sont pas atteints pour 7 des 8 objectifs principaux (tableaux 5 et 6). Si Robison se réjouit de comparaisons avantageuses avec l'Angleterre (prise en charge aux urgences), l'opposition lui rappelle que les pourcentages représentent des cas concrets de patients attendant plus de trois mois, pour consulter un spécialiste (triplement en quatre ans) ou pour une opération (120 000 personnes), ce qui conduit certains à ne pas honorer leur rendez-vous, notamment en zone défavorisée ${ }^{22}$. En conséquence, son successeur, Jeane Freeman, a accordé une rallonge budgétaire à des health boards sommés de rendre des comptes détaillés ${ }^{23}$. Mais elle préfère optimiser les structures publiques au lieu de recourir à des sociétés privées. Elle a reporté l'application des objectifs à 2021.

15 Ensuite, les patients doivent donner leur avis. Dès les débuts de la dévolution, chaque décision de fermeture de services hospitaliers a suscité un tollé, sous forme de manifestations, pétitions, et même de l'élection à Holyrood en 2003 d'un médecin impliqué dans ces mouvements. McConnell a instauré un Scottish health council, consulté par les health boards. Mais c'est en fait le gouvernement écossais qui est tenu pour responsable. Le SNP, élu en 2007 à l'issue d'une campagne ciblant les services d'urgence d'Ayr et de Monkland, a subi les conséquences des fermetures ultérieures. Dans ce contexte, le gouvernement Salmond a repris une proposition formulée en 2002 par un député travailliste, l'élection directe de citoyens aux conseils des health boards. Cette réforme, adoptée à l'unanimité en 2009, était censée refléter l'attitude des citoyens écossais, « propriétaires » de leur NHS, par opposition aux consommateurs anglais ${ }^{24}$. Mais lors des élections de 2010, et en dépit de l'abaissement de la majorité électorale à 16 ans, la participation est restée faible (Fife $14 \%$, Dumfries \& Galloway 22,6 \%) ${ }^{25}$. Par conséquent, en novembre 2013, Alex Neil, ministre de la Santé, est revenu à la nomination de citoyens après avis d'une commission indépendante, incitée à stimuler la diversité des candidatures (sur le modèle de Grampian et Lothian). Le gouvernement Sturgeon, ainsi que plusieurs conseils, ont par ailleurs mis en place des forums de citoyens.

16 Enfin, l'opinion des Écossais est mitigée ${ }^{26}$. Après 10 ans de pouvoir du SNP, des proportions équivalentes d'Écossais (45\%) se déclarent satisfaits et mécontents de sa politique. En dépit des critiques, le SNP a rattrapé son retard sur le Parti travailliste sur

Revue Française de Civilisation Britannique, XXIV-3 | 2019 
un enjeu essentiel. Qui plus est, en 2014, il a convaincu nombre d'électeurs d'opter pour l'indépendance afin de protéger le NHS écossais des influences anglaises.

Cependant, la majorité des Écossais ne se distingue guère des Anglais, s'agissant des attentes à l'égard du NHS ou de la priorité financière à lui accorder ${ }^{27}$. Si deux-tiers des Écossais approuvent le principe de dévolution de la santé, seule la moitié en accepte les conséquences, qu'il s'agisse de financement par le Parlement écossais ou de la possibilité de politiques divergentes telle la suppression du ticket modérateur sur les médicaments, présentée comme l'une des innovations permises par la dévolution.

\section{La dévolution, source d'innovations?}

Les élus écossais ont tenté de modifier les comportements individuels, toute amélioration de la santé publique devant alléger les dépenses du NHS. Ils ont ciblé les médicaments, l'alimentation et les addictions.

\section{Le coût des soins}

La gratuité des services publics, en conférant des droits identiques à tous les membres de la société, est censée atténuer les inégalités d'accès. Au sein du NHS, avant 2007, elle avait été restaurée pour les examens dentaires et de vision. En marge de celui-ci, elle avait été accordée aux personnes âgées dépendantes afin de subvenir à leurs frais de soins personnels. Le gouvernement SNP a supprimé les frais de parking à l'hôpital (à l'exception des trois entités construites et entretenues par des sociétés privées dans le cadre de partenariats public-privé) en 2008, avant d'abolir progressivement le ticket modérateur sur les médicaments.

$90 \%$ des médicaments étaient déjà gratuits, mais cela ne bénéficiait qu'à $50 \%$ des patients (personnes âgées, enfants, certains malades chroniques). Dès 2006, le SNP avait soutenu une proposition de loi du Scottish Socialist Party (SSP), visant la généralisation de la gratuité, mais le gouvernement McConnell craignait une explosion de la demande ${ }^{28}$. L'année suivante, Sturgeon, ministre de la Santé, a indiqué vouloir abroger cette taxe sur la mauvaise santé, un élargissement des catégories bénéficiaires risquant de créer de nouvelles inégalités et d'entraîner des frais de gestion ${ }^{29}$. Elle a su convaincre les Travaillistes en évoquant les principes fondateurs du NHS en 1948, surmontant leurs réticences envers l'inclusion des classes moyennes ${ }^{30}$.

21 Or, le coût de cette abrogation du ticket modérateur ayant fait un bond de $25 \%$ de 2011 à 2018, des professionnels de santé ont réclamé sa restauration, pour les personnes aisées, ou pour les médicaments disponibles sans ordonnance ${ }^{31}$. Mais le gouvernement écossais a défendu cette mesure emblématique de la dévolution (le ticket atteignant 9 livres en Angleterre), qui évitait un afflux vers des hôpitaux surchargés. Il pouvait compter sur un consensus, avec les Travaillistes (peu désireux de rouvrir le débat qu'ils avaient amorcé en 2012) et les Conservateurs (auxquels Ruth Davidson avait imposé un revirement).

\section{L'alimentation des enfants}

S'agissant des nourrissons, le Parlement écossais a adopté en 2004 une loi visant à stimuler l'allaitement maternel, notamment dans les milieux défavorisés, où la pression 
sociale à l'encontre de ces pratiques était la plus forte, comme l'avait remarqué Elaine Smith, députée travailliste à l'origine du texte ${ }^{32}$. Le gouvernement McConnell ne s'y est pas opposé, malgré ses réserves quant à la méthode (empêcher d'interdire l'allaitement maternel dans les lieux publics), en raison de l'objectif de santé publique poursuivi. Seuls les Conservateurs estimaient que ce dernier ne devait pas empiéter sur la liberté individuelle.

Quant aux enfants, en 2003, le gouvernement McConnell a axé sa politique sur la qualité des repas servis dans les cantines scolaires, avec des objectifs nutritionnels et éducatifs. Il a introduit certains aliments (fruits, eau, lait), tout en révisant les normes et les portions, ainsi que sur le contenu des distributeurs automatiques (programme Hungry for Success). Confronté à des habitudes alimentaires tenaces, John Swinney, ministre de l'Éducation (SNP), a proposé en 2018 un nouveau plan, pour réduire le sucre, favoriser les fruits et légumes.

Le SNP soutenait par ailleurs la gratuité des repas, afin d'encourager les enfants à déjeuner à la cantine, notamment les plus modestes, souvent stigmatisés. La première tentative, portée par le SSP, a échoué en 2002, au regard de son coût ${ }^{33}$. Arrivé au pouvoir, le SNP a ciblé les trois premières années d'école primaire, d'abord dans cinq collectivités territoriales au niveau de vie inférieur à la moyenne (Glasgow, West Dunbartonshire, East Ayrshire, Fife, Borders), puis dans toute l'Écosse ${ }^{34}$. Dès 2015-2016, parmi les enfants en école primaire, plus de $80 \%$ prenaient leurs repas à la cantine lors des trois premières années, contre $50 \%$ les années ultérieures ${ }^{35}$. Seuls les Conservateurs sont restés réticents.

Cependant, les besoins se sont multipliés en raison de la réforme britannique des prestations sociales qui, en entraînant des retards, des réductions, voire des suspensions dans les versements, pousse nombre de familles à se tourner vers les banques alimentaires. En 2018, North Lanarkshire (gérée par les Travaillistes) a annoncé l'ouverture des cantines pendant les vacances scolaires tandis que Glasgow (dirigée par le SNP) a étendu la gratuité à la quatrième année de scolarité ${ }^{36}$. En outre, grâce aux compétences transférées par le Scotland Act de 2016, le Parlement écossais a voté en 2018 à l'unanimité une loi portant création d'un service écossais de Sécurité Sociale, afin de revaloriser certaines prestations et de mettre un terme aux sanctions arbitraires, ce qui devrait bénéficier aux enfants vivant dans la pauvreté (15\%, tableau 3$)^{37}$.

\section{La lutte contre les addictions}

Les gouvernements écossais successifs ont mené des politiques volontaristes face à des lobbys puissants.

S'agissant de lutte contre l'alcoolisme, après avoir obtenu le droit de déterminer la limite acceptable pour les conducteurs (Scotland Act de 2012), les dirigeants écossais ont abaissé cette dernière, de 0,8 à $0,5 \mathrm{~g}$ par litre de sang, par une loi votée en 2014 à l'unanimité ${ }^{38}$. Ils ont bravé l'opposition des gérants de public houses, lesquels continuent à rendre cette loi responsable de leur déclin.

Par ailleurs, ils ont cherché à agir sur l'offre. En 2005, la coalition de McConnell a imposé la santé publique parmi les critères à respecter pour les détenteurs de licences de consommation sur place, sans parvenir à convaincre ses opposants ${ }^{39}$. Le SNP voulait agir sur les prix pratiqués sur les ventes à emporter. En 2010, Sturgeon, ministre de la Santé, est simplement parvenue à interdire les promotions liées à la quantité, par une loi votée à 
l'unanimitét ${ }^{40}$. En 2012, elle a réuni une large majorité autour de l'objectif poursuivi depuis 2009, un prix minimum. Les Conservateurs ont toléré une période d'essai de cinq ans; les Travaillistes ont préféré l'abstention, faute de pouvoir infliger une taxe spéciale aux supermarchés ${ }^{41}$. Mais l'association des producteurs de whisky (Scottish Whisky association) s'est tournée vers la justice, invoquant le droit communautaire, ainsi que l'équilibre entre santé publique et liberté du commerce. La Cour Suprême a saisi la Cour de Justice de l'UE (laquelle a validé les possibilités de restrictions, proportionnées aux objectifs, pour des raisons de santé publique), avant de conclure, en novembre 2017, que le gouvernement écossais avait un objectif clair, la lutte contre l'alcoolisme, et une méthode, ciblant les alcools les moins chers, prisés par les plus démunis ${ }^{42}$. Le $1^{\text {er }}$ mai 2018, lors de l'entrée en vigueur de la loi, Sturgeon, Premier ministre, a fixé le prix minimum à 50 pence par degré d'alcool, ce qui concerne $70 \%$ des alcools vendus ${ }^{43}$. La ministre Robison anticipait des effets sur la santé et les finances publiques ${ }^{44}$, voire un modèle pour les îles britanniques.

Quant à la lutte contre le tabagisme, en 2005, sous le gouvernement McConnell, le Parlement écossais a interdit l'usage de la cigarette dans les lieux publics fermés, se félicitant de montrer la voie au reste de la Grande-Bretagne ${ }^{45}$. En 2010, sous le gouvernement Salmond, il a prohibé certaines modalités de vente (dans les distributeurs automatiques, ou à des mineurs de moins de 18 ans) et de promotion dans les magasins, tout en créant un registre des distributeurs ${ }^{46}$. En 2016, nombre de ces mesures ont été étendues au vapotage. Seuls les Conservateurs ont rejeté ces textes, trop contraignants pour les entreprises. En 2018, le gouvernement écossais entend faire des jeunes une génération sans tabac à l'horizon 2034.

Les députés écossais, fiers de leur bilan, craignent les effets du Brexit ${ }^{47}$. La sortie des dispositifs communautaires pourrait remettre en cause la disponibilité des médicaments (et l'accès aux traitements innovants), tandis que le gouvernement britannique pourrait être tenté de conclure des accords de libre échange autorisant des sociétés privées à poursuivre le gouvernement écossais à cause de ses politiques de santé publique. Cela ne pourrait que contrarier l'objectif d'amélioration de la santé des Écossais.

\section{La dévolution, moyen d'amélioration de la santé des Écossais?}

31 L'espérance de vie, indicateur synthétique, a progressé de 25 ans en un siècle, pour s'établir à 81,4 ans pour les femmes, 77,4 ans pour les hommes, tout en restant inférieure de deux ans à celle des autres nations britanniques. Elle varie de plus de sept ans pour les hommes selon les collectivités territoriales (tableau 1). À l'intérieur de celles-ci, elle fluctue de 67 ans dans les quartiers défavorisés de Glasgow, à plus de 81 ans dans les quartiers aisés de la collectivité voisine d'East Dunbartonshire. La fourchette est moindre, mais significative, s'agissant des femmes. Si la santé de la population écossaise s'est améliorée, notamment grâce aux choix politiques locaux, les écarts avec les pays occidentaux n'ont pas disparu.

\section{Une mortalité relativement élevée}

Entre 1945-1951 et 2001, le taux de mortalité infantile (au cours de la première année de vie) a été divisé par dix (de 53,2 à 5,8 pour mille), tandis que le taux de mortalité des 
hommes a été divisé par deux dans la tranche $45-54$ ans (de 10,6 pour mille à 5,3 en 2001), en diminuant d'un-tiers au-delà ${ }^{48}$.

Cependant, à la fin des années soixante-dix, l'Écosse se situait au $31^{\mathrm{e}}$ rang sur 33 pays développés pour la mortalité, au $12^{\mathrm{e}}$ rang pour le taux de mortalité infantile ( $7^{\mathrm{e}}$ en 1950$)^{49}$. En 2010, le taux de mortalité restait supérieur à la moyenne européenne, de $21 \%$ chez les hommes et de $30 \%$ chez les femmes ${ }^{50}$. Mais la chute du taux de mortalité infantile avait été plus rapide qu'en Angleterre.

Ce constat valait pour les principaux facteurs de mortalité. Les maladies cardiovasculaires, premières causes de décès, étaient supérieures de 39 \% à la moyenne européenne (contre 73 \% en 1955 néanmoins). La mortalité liée aux maladies chroniques du foie était la plus élevée d'Europe, et poursuivait sa progression, constituant le deuxième facteur de mortalité des personnes de moins de 65 ans.

Qui plus est, l'état de santé fluctue en fonction du milieu social. En 1998, les problèmes cardiovasculaires étaient deux fois plus fréquents chez les ouvriers que chez les cadres supérieurs et professions libérales ${ }^{51}$. Leurs conséquences étaient plus graves, avec un écart de plus de 4,5 dans le taux de mortalité, en 1992 comme en $2001^{52}$. La ville de Glasgow, l'une des plus pauvres du Royaume-Uni, cumule les difficultés sociales ${ }^{53}$. Les taux de mortalité prématurée (avant 75 ans) due aux maladies du cœur et aux cancers dépassent la moyenne écossaise (de 20\%), mais aussi ceux des autres grandes villes (tableau 2).

\section{Des comportements à risque}

En ce qui concerne l'alimentation, elle est plus déséquilibrée en Écosse ${ }^{54}$. De plus, on constate des écarts de 1 à 2 , selon le revenu, dans la propension à consommer régulièrement des sodas, des frites, des fruits frais, ou à ajouter du sel ${ }^{55}$. En 2016, seuls $20 \%$ des adultes et $12 \%$ des enfants mangeaient cinq fruits et légumes par jour, ces proportions restant stables depuis 10 ans.

La proportion de nourrissons bénéficiant de l'allaitement maternel à 6-8 semaines est passée de $36,5 \%$ en 2004 à $41 \%$ en 2017. Mais si plus de la moitié des enfants sont allaités à un moment, le taux varie fortement selon le niveau de vie de la collectivité territoriale (écarts de 2,5) (tableau 3).

L'obésité se développe, affectant $30 \%$ des adultes et $15 \%$ des enfants (cette proportion étant supérieure de moitié dans les milieux défavorisés). L'obésité sévère (IMC supérieur à 40 ) concerne $4 \%$ de la population écossaise, taux supérieur à celui des nations voisines mais dont la progression relative devrait ralentir.

S'agissant des addictions, le tabac est responsable d'un-cinquième des décès et de 128000 admissions à l'hôpital chaque année. Si l'alcool est la cause d'un décès sur quinze et de 36 000 hospitalisations, il a de multiples effets sociaux : il est cité dans la majorité des délits commis par les jeunes, dans un tiers des divorces et affecte 65000 enfants élevés par un parent souffrant de cette dépendance ${ }^{56}$.

Certes, les tendances s'améliorent. Ainsi, la proportion de fumeurs est passée de $50 \%$ des hommes en 1980 à un tiers en 1998, puis un quart en 2013 ${ }^{57}$. Entre 2003 et 2013, les syndromes coronariens aigus ont davantage chuté en Écosse qu'en Angleterre ${ }^{58}$. Quant à la consommation d'alcool par habitant, dans les années 2000, elle était supérieure de $25 \%$ 
à celle de l'Angleterre, se situant au huitième rang mondial. Elle a baissé entre 2003 et 2013, passant de 16 à 12 unités par adulte et par semaine ${ }^{59}$.

41 Mais l'écart dans la proportion de fumeurs selon le niveau de vie s'est creusé. Les admissions à l'hôpital dues à l'alcool sont huit fois supérieures dans les quartiers défavorisés par rapport aux zones plus favorisées. C'est à nouveau à Glasgow que la mortalité imputable à l'alcool ou au tabac est la plus élevée (avec un taux 2,5 fois supérieur aux Borders pour l'alcool) (tableau 2).

Les politiques de prévention sont diversement appliquées selon le milieu social. La proportion d'écoliers souffrant de caries dentaires variait de 1 à 3 dans les années soixante-dix, de 1 à 2 en $2016^{60}$. De plus, les personnes résidant dans les territoires démunis sont proportionnellement moins nombreuses à bénéficier des programmes de dépistage (tableau 3). Ainsi, les cancers sont plus souvent détectés à un stade avancé.

\section{Une population vieillissante}

La hausse de l'espérance de vie, associée à la chute des naissances (le taux de fertilité, 1,7 enfant par femme, étant inférieur au seuil de renouvellement des générations) a entrâné un vieillissement de la population. Ainsi, la proportion de personnes de plus de 65 ans, passée de $5 \%$ à $18 \%$ au xxe siècle, devrait continuer à croître d'ici 2039, en particulier en milieu rural ${ }^{61}$. Cela va entraîner une augmentation de la demande de soins, l'espérance de vie en bonne santé (sans maladie chronique ou invalidante) étant de 60,3 ans pour les hommes et 62,6 pour les femmes (avec des écarts de 10 ans selon le lieu). La demande sera forte dans les régions rurales périphériques comptant déjà plus de personnes âgées que la moyenne (tableau 3) et dans les régions centrales urbaines, où les problèmes de santé sont plus fréquents. Le financement posera problème, le ratio inactifs (enfants et retraités)/actifs devant passer de $58 \%$ à $67 \%$.

Ce vieillissement est plus marqué en Écosse, où l'âge médian, 41,9 ans en 2016, est supérieur de deux ans à la moyenne britannique. Il s'est accompagné d'un déclin de la population, à partir des années soixante-dix, alors que les trois autres nations poursuivaient leur croissance. Dans les années 2000, la tendance s'est inversée, grâce à l'arrivée de ressortissants des États d'Europe centrale qui ont intégré l'UE en 2004 (le solde naturel restant faible ou négatif). À l'avenir, seule l'immigration peut maintenir cette dynamique, à la différence du reste du Royaume-Uni. Afin d'éviter les conséquences démographiques dramatiques d'une fermeture des frontières consécutive au Brexit, le gouvernement SNP réclame au gouvernement britannique la dévolution de la politique d'immigration, ou, à défaut, une prise en compte des besoins de l'Écosse. Ces immigrés, considérés comme un atout, doivent stimuler la croissance et les recettes fiscales (leur contribution annuelle excédant 10000 livres $^{62}$ ), seuls moyens de maintenir un service public de santé de qualité.

\section{Conclusion}

La santé des Écossais s'est améliorée, suivant une tendance générale dans les pays occidentaux, mais aussi grâce à l'attention accordée par les dirigeants politiques aux besoins locaux. Le maintien de structures publiques, un des fondements de la dévolution, n'a jamais été remis en cause, sans pour autant faire la preuve de son efficacité au regard 
des listes d'attentes ou de la réduction des inégalités, tant pour la prévention que pour les soins. Les politiques publiques ont permis aux élus de distinguer l'Écosse de l'Angleterre, en faisant un modèle en Europe et dans le monde, rompant avec l'image antérieure, tout en alourdissant les coûts.

Cependant, en dépit de cette continuité, les politiques de santé n'échappent pas à l'instrumentalisation politique. Le SNP, en cultivant son image sociale, a ravi au Parti travailliste sa position dominante. Il est parvenu à articuler l'austérité pratiquée par le gouvernement britannique aux questions institutionnelles, en faisant un enjeu du référendum d'autodétermination de 2014, puis du Brexit. Il contraint ses adversaires à réagir sur ce terrain, les Conservateurs se démarquant de certaines politiques menées par le gouvernement de Theresa May, tandis que les Travaillistes prônent une hausse des dépenses face à un SNP obnubilé par son objectif indépendantiste. Mais, au-delà des polémiques, le gouvernement Sturgeon est confronté à une situation qui se dégrade, qu'il s'agisse des finances publiques (Audit Scotland, organisme de contrôle des comptes publics, constatant que NHS Scotland n'est pas dans une situation viable) ou de l'espérance de vie (qui régresse pour la première fois en trente-cinq ans). Le secteur de la santé, sur le point de représenter la moitié du budget écossais, va exiger des choix de la part d'un gouvernement minoritaire et privé de nombreux leviers d'action dans le cadre de la dévolution du pouvoir.

Edwige Camp-Pietrain est Professeur de civilisation britannique à l'Université Polytechnique des Hauts-de-France (Valenciennes). Membre du laboratoire CALHISTE EA 4343, elle est spécialiste de la vie politique, des institutions et des politiques publiques dans l'Écosse contemporaine.

\section{BIBLIOGRAPHIE}

AUDITOR GENERAL (AG), Tackling Waiting Times in the NHS in Scotland, Édimbourg, Audit Scotland, 2006.

AG, Health Inequalities in Scotland, Édimbourg, Audit Scotland, 2012.

AG, NHS in Scotland, Édimbourg, Audit Scotland, octobre 2016.

AG, NHS in Scotland, Édimbourg, Audit Scotland, octobre 2017.

BEVAN Gwyn, KARANIKolos Marina, EXLEY Jo, NOLTE Eileen, CONNOlly Sheelah, MAYs Nicholas, The Four Health Systems of the United Kingdom : How do they Compare?, Londres, Nuffield Trust, 2014. BROoKs Libby, «Council Plans Free School Meals All Year to Tackle 'Holiday Hunger' », The Guardian, 16 février 2018.

BROOKS Richard, « Scotland's Health » in Ingram Keith, Love James (dir), Understanding the Scottish Economy, Oxford, Martin Robertson, 1983, p. 107-116.

BRUCE Allan, FORBES Tom, «From Competition to Collaboration in the Delivery of Health Care : Implementing Change in Scotland », Scottish Affairs, $\mathrm{n}^{\circ}$ 34, 2001, p. 107-124. 
CHURCH Jenny (dir), Regional Trends, Londres, HMSO, 1994.

DAYAN Mark, EDWARDS Nigel, Learning from Scotland's NHS, Londres, Nuffield Trust, juillet 2017. DoDDS Anneliese, « The Politicisation of Trade in Health and Education Services : Black and White Divisions over a 'Grey' Area », Scottish Affairs, n46, 2014, p. 56-75.

GREER Scott, Territorial Politics and Health Policy, Manchester, Manchester University Press, 2004. GREER Scott, WILSON Iain, DONNELLY Peter, «The Wages of Continuity : Health Policy under the SNP », Scottish Affairs, vol. 25, n¹, 2016, p. 28-44.

GREER Scott, WILSON Iain, STEWART Ellen, DONNELLy Peter, Health Board Elections and Alternative Pilots : Final Report of the Statutory Evaluation, Édimbourg, Scottish Government, 2012.

HAMILTON David, « Health and Health Care in West Central Scotland », Scottish Government Yearbook 1978, p. 77-87.

HAMILTON David, « Health », in MACDONALD Margo (dir), The Radical Approach. Papers on an Independent Scotland, Édimbourg, Palingenesis Press, 1976, p. 64-75.

HEENAN Deirdre, BIRRELL Derek, The Integration of Health and Social Care in the UK, Londres, Palgrave Macmillan, 2018.

HOUSE OF COMMONS (HC), Hansard, 23 mai 2018

HC, Scottish Affairs Committee, Demography of Scotland and the Implications for Devolution, Londres, HC 82, 2016.

HUNTER D., " The Reorganised Health Service », Scottish Government Yearbook, 1977, p. 26-37.

KEATING Michael, LINEIRA Robert, « Getting to a Wealthier and Fairer Scotland ", in KEATING Michael (dir), A Wealthier, Fairer Scotland, Édimbourg, Edinburgh University Press, 2017, p. 129-145.

LUDBROoK Anne, THEODOSSIOU Ioannis, GEROVA Vania, « Health and Deprivation » in ERMISCH John, WRIGHT Robert (dir), Changing Scotland, Bristol, Policy Press, 2005, p. 99-111.

MCADAMS Rachel, Evaluating Universal Infant Free School Meals, Scotland, Édimbourg, NHS Health Scotland, octobre 2016 .

MCARDLE Helen, « Free Prescription 'Save NHS Money' Despite Soaring Bill for Ageing and Obesity », The Herald, 5 octobre 2017, p. 6.

MCCRONE David, The New Sociology of Scotland, Londres, Sage, 2017.

MCKENDRICK John, « What is Life Like for People Experiencing Poverty? », in MCKENDRICK John, MOONEY Gerry, sCOTT Gill, DICKIE John, MCHARDY Fiona (dir), Poverty in Scotland 2016. Tools for Transformation, Londres, CPAG, 2016, p. 113-127.

MCLEAN Joanne, CHRISTIE Joanne, GRAY Lindsay, Scottish Health Survey, Édimbourg, Scottish Government, 2016.

MCTAVISH Duncan, « Scottish and English Health Policy from 1948 to the 1973 Reforms :

Management Through a UK Prism? », Scottish Affairs, n 51, printemps 2005, p. 59-78.

MILNE Robin, «Competitive Tendering of NHS Hotel Services in Scotland », Scottish Affairs, $\mathrm{n}^{\circ} 3$, 1993, p. 135-146.

NATIONAL RECORDS OF SCOTLAND, Life Expectancy for Areas within Scotland 2013-2015, Édimbourg, 2016. 
ORMSTON Rachel, cURTICE John, « Attitudes Towards a 'British Institution' : Comparing Public Views of the NHS in England and Scotland», Scottish Affairs, $n^{\circ} 61,2007$, p. 50-73.

PATERSON Lindsay, BECHHOFER Frank, MCCRONE David, Living in Scotland. Social and Economic Change since 1980, Édimbourg, Edinburgh University Press, 2004.

ROBERTSON Tony, MARSDEN Sara, KAPILASHRAmi Anuj, « A Public Health Politics that is People's Health ", in HASSAN Gerry, BARROW Simon (dir), A Nation Changed? The SNP and Scotland Ten Years On, Édimbourg, Luath Press, 2017, p. 124-131.

scottish government (SG), Scotland's Place in Europe. People, Investment, Jobs, Édimbourg, 2018.

SG, Brexit, Protecting What Matters. What's at Stake for Individuals, Édimbourg, 2018.

scотPно, Health and Well-Being Profiles 2015. Scotland Overview Report, Édimbourg, NHS, 2015.

SCOTTISH PARLIAMENT (SP), Official Report (OR), 20 juin 2002.

SP, OR, 18 novembre 2004.

SP, OR, 30 juin 2005.

SP, OR, 16 novembre 2005.

SP, OR, 25 janvier 2006.

SP, OR, 5 décembre 2007.

SP, OR, 12 mars 2009.

SP, OR, 27 janvier 2010.

SP, OR, 18 novembre 2010.

SP, OR, 24 février 2011.

SP, OR, 10 novembre 2011.

SP, OR, 24 mai 2012.

SP, OR, 19 février 2014

SP, OR, 19 août 2014.

SP, OR, 18 novembre 2014.

SP, OR, $1^{\text {er }}$ novembre 2017.

SP, OR, 21 novembre 2017.

SP, OR, 17 avril 2018.

SP, OR, 25 avril 2018.

SP, OR, 2 mai 2018.

SP, OR, 26 juin 2018.

SP, OR, 4 octobre 2018.

SP, OR, 24 octobre 2018.

SP, Culture, Tourism, Europe and External Relations Committee, Brexit-What Scotland Thinks, Édimbourg, SP 64, 2017.

SP, Health and Sport Committee (HSC), Stage 1 Report on the Alcohol (Minimum Pricing) (Scotland) Bill, Édimbourg, 2012. 
SP, HSC, OR, 6 mars 2018.

SP, HSC, OR, 20 mars 2018.

SUPREME COURT, Scotch Whisky Association and others v. Lord Advocate and Another, Londres, UKSC 76, 15 novembre 2017.

wOods Kevin, « Scotland's Changing Health System », in wOODs Kevin, CARTER David (dir), Scotland's Health and Health Services, Londres, Stationery Office, 2003, p. 1-29.

\section{ANNEXES}

\section{Annexes}

Tableau 1: Espérance de vie à la naissance dans les collectivités territoriales en 2011-2015

\begin{tabular}{|c|c|c|c|c|c|c|}
\hline & Hommes & & & Femmes & & \\
\hline $\begin{array}{l}\text { Collectivité } \\
\text { territoriale }\end{array}$ & Écosse & $\begin{array}{l}\text { Zones les } \\
\text { plus riches }\end{array}$ & $\begin{array}{l}\text { Zones les } \\
\text { plus pauvres }\end{array}$ & Écosse & $\begin{array}{l}\text { Zones les } \\
\text { plus riches }\end{array}$ & $\begin{array}{l}\text { Zones les } \\
\text { plus pauvres }\end{array}$ \\
\hline Aberdeen & 76,6 & 77,7 & 71,4 & 80,9 & 81,6 & 77,9 \\
\hline Aberdeenshire & 79,2 & 79,8 & 75,6 & 82,2 & 82,6 & 79,9 \\
\hline Angus & 78,6 & 79,3 & 74 & 81,9 & 82,2 & 78,9 \\
\hline Argyll\&Bute & 78,2 & 78,8 & 72,8 & 82,2 & 82,4 & 78,3 \\
\hline Édimbourg & 78 & 79,1 & 70,5 & 82,2 & 83,2 & 76 \\
\hline Clackmannanshire & 76,9 & 77,6 & 73,1 & 80,1 & 81 & 75,5 \\
\hline Dumfries\&Galloway & 78,1 & 78,7 & 74,2 & 81,3 & 82 & 78,4 \\
\hline Dundee City & 75,1 & 76 & 68,8 & 80,1 & 80,3 & 76,3 \\
\hline East Ayrshire & 76,1 & 76,7 & 71,9 & 79,4 & 80,3 & 76,1 \\
\hline East Dunbartonshire & 80,5 & 81,2 & 75 & 83,5 & 84,5 & 79,2 \\
\hline East Lothian & 78,4 & 78,7 & 75,8 & 82,5 & 82,4 & 79,9 \\
\hline East Renfrewshire & 79,3 & 80,9 & 72,7 & 83,4 & 84 & 78,6 \\
\hline Falkirk & 77,4 & 78,1 & 71,7 & 80,9 & 81,1 & 78,8 \\
\hline Fife & 77,7 & 78,3 & 72,9 & 81,5 & 81,8 & 78,4 \\
\hline Glasgow & 73,4 & 74,2 & 67,8 & 78,8 & 79,4 & 74,8 \\
\hline Highland & 77,9 & 78,6 & 72,1 & 82,6 & 83,1 & 77,9 \\
\hline
\end{tabular}




\begin{tabular}{|c|c|c|c|c|c|c|}
\hline Inverclyde & 75,4 & 76,3 & 68,5 & 80,4 & 80,9 & 77 \\
\hline Midlothian & 77,3 & 77,8 & 74,2 & 81,5 & 82 & 78,7 \\
\hline Moray & 78,7 & 78,7 & 75 & 81,7 & 82 & 79,4 \\
\hline Hébrides extérieures & 76,7 & 76,6 & 77,2 & 82,9 & 81,1 & 84,4 \\
\hline North Ayrshire & 76,1 & 77,2 & 69,7 & 80,8 & 81,4 & 77,6 \\
\hline North Lanarkshire & 75,3 & 76,2 & 70,2 & 79,6 & 80,1 & 75,7 \\
\hline Orcades & 78,8 & 79,8 & 75,2 & 82,8 & 83 & 80,2 \\
\hline Perth \& Kinross & 79,8 & 80,4 & 73,7 & 82,6 & 83,1 & 79,5 \\
\hline Renfrewshire & 76,3 & 77,1 & 69,9 & 80,6 & 81,2 & 75,9 \\
\hline Scottish Borders & 78,8 & 79,5 & 75,5 & 82,5 & 82,8 & 79,6 \\
\hline Shetlands & 77,6 & 78,3 & 71,8 & 81,9 & 82,4 & 79,9 \\
\hline South Ayrshire & 77,7 & 78,6 & 72,9 & 81 & 81,3 & 78,5 \\
\hline South Lanarkshire & 77 & 77,7 & 71,1 & 80,8 & 81,2 & 77,6 \\
\hline Stirling & 78,5 & 79,7 & 72,2 & 82 & 82,8 & 77,5 \\
\hline $\begin{array}{l}\text { West } \\
\text { Dunbartonshire }\end{array}$ & 74,8 & 75,1 & 69,2 & 78,7 & 79,2 & 77,1 \\
\hline West Lothian & 78,2 & 78,3 & 74,6 & 80,8 & 80,8 & 78,5 \\
\hline Écosse & 77,1 & 78,1 & 70,8 & 81,1 & 81,8 & 76,8 \\
\hline
\end{tabular}

Source : NATIONAL RECORDS OF SCOTLAND, Life Expectancy for Areas within Scotland 2013-2015, Édimbourg, 2016, tableau 1.

Tableau 2 : Espérance de vie (en années) et mortalité anticipée (sur 100000 personnes) dans les health boards en 2010-2013

\begin{tabular}{|l|l|l|l|l|l|}
\hline & $\begin{array}{l}\text { Espérance vie } \\
\text { masculine }\end{array}$ & $\begin{array}{l}\text { Mortalité } \\
\text { anticipée pour } \\
\text { maladies cœur }\end{array}$ & $\begin{array}{l}\text { Mortalité } \\
\text { anticipée pour } \\
\text { cancer }\end{array}$ & $\begin{array}{l}\text { Mortalité } \\
\text { liée au tabac }\end{array}$ & $\begin{array}{l}\text { Mortalité } \\
\text { liée à alcool }\end{array}$ \\
\hline $\begin{array}{l}\text { Ayrshire \& } \\
\text { Arran }\end{array}$ & 76,5 & 67,3 & 171,7 & 333 & 22,9 \\
\hline Borders & 78,7 & 43 & 134,2 & 279,6 & 13,4 \\
\hline
\end{tabular}




\begin{tabular}{|l|l|l|l|l|l|}
\hline $\begin{array}{l}\text { Dumfries } \\
\text { Galloway }\end{array}$ & 77,5 & 52,2 & 156,2 & 306,2 & 15,2 \\
\hline Fife & 77 & 54,5 & 167,3 & 333 & 21,7 \\
\hline Forth Valley & 77,4 & 58,1 & 170,1 & 316,2 & 19,9 \\
\hline Grampian & 78 & 48,4 & 155,4 & 281,8 & 16,3 \\
\hline $\begin{array}{l}\text { Greater } \\
\text { Glasgow } \\
\text { Clyde }\end{array}$ & 74,5 & 72,1 & 202,8 & 389,7 & 33 \\
\hline Highland & 77,2 & 48,9 & 160,5 & 265 & 23,5 \\
\hline Lanarkshire & 75,6 & 72,4 & 184,4 & 363,2 & 28,8 \\
\hline Lothian & 77,5 & 54,7 & 170,9 & 303,1 & 19,4 \\
\hline Orcades & 79,7 & 39,3 & 121,5 & 248,6 & 25,1 \\
\hline Shetland & 77,4 & 51 & 173,1 & 275,2 & 15,1 \\
\hline Tayside & 77,3 & 56,2 & 155,4 & 288,2 & 22,7 \\
\hline Écostérieures & 76,4 & 60,1 & 173,4 & 325,9 & 23,8 \\
\hline
\end{tabular}

Source : sсотрно, Health and Well-Being Profiles 2015. Scotland Overview Report, Édimbourg, NHS, 2015, pp. 30-43.

Tableau 3 : Quelques caractéristiques de la population des health boards en 2010-2013 (\%)

\begin{tabular}{|l|l|l|l|l|l|}
\hline & $\begin{array}{l}\text { Personnes } \\
\text { de plus de } \\
75 \text { ans } \\
\text { recevant } \\
\text { allocations liées } \\
\text { à l'absence } \\
\text { d'emploi }\end{array}$ & $\begin{array}{l}\text { Enfants } \\
\text { pauvres }\end{array}$ & $\begin{array}{l}\text { Enfants de mois } \\
\text { bénéficiant } \\
\text { allaitement } \\
\text { maternel exclusif }\end{array}$ & $\begin{array}{l}\text { Prévention } \\
\text { cancer intestin } \\
\text { (personnes de 50 } \\
\text { às) }\end{array}$ \\
\hline $\begin{array}{l}\text { Ayrshire \& } \\
\text { Arran }\end{array}$ & 9,3 & 16 & 19 & 18,1 & 55,2 \\
\hline Borders & 10,2 & 10 & 10,9 & 32,5 & 60,4 \\
\hline $\begin{array}{l}\text { Dumfries \& } \\
\text { Galloway }\end{array}$ & 10,8 & 12 & 13,9 & 23,1 & 58,3 \\
\hline Fife & 8,5 & 13,4 & 16,4 & 24,9 & 56,1 \\
\hline
\end{tabular}




\begin{tabular}{|l|l|l|l|l|l|}
\hline Forth Valley & 7,9 & 12,6 & 14,6 & 23 & 54,6 \\
\hline Grampian & 7,6 & 7,3 & 8,5 & 33 & 60,5 \\
\hline $\begin{array}{l}\text { Greater } \\
\text { Glasgow \& } \\
\text { Clyde }\end{array}$ & 7,6 & 16,9 & 20,4 & 23,3 & 51,5 \\
\hline Highland & 9,5 & 9,9 & 11,1 & 31,2 & 60,4 \\
\hline Lanarkshire & 7,5 & 15,3 & 16,7 & 17,8 & 49 \\
\hline Lothian & 7,2 & 10,8 & 13,7 & 34,8 & 53,5 \\
\hline Orcades & 9,7 & 6,6 & 6,1 & 41,2 & 62,4 \\
\hline Shetland & 7,9 & 6,5 & 6 & 41,9 & 63,8 \\
\hline Tayside & 9,5 & 12,3 & 14,1 & 26 & 60 \\
\hline Hébrides \\
extérieures
\end{tabular}

Source : sсотРно, Health and Well-Being Profiles 2015. Scotland Overview Report, Édimbourg, NHS, 2015, pp. 30-43.

Tableau 4 : Le personnel des health boards en 2016

\begin{tabular}{|l|l|l|l|l|}
\hline & $\begin{array}{l}\text { Taux } \\
\text { d'absentéisme } \\
\text { pour maladie }\end{array}$ & $\begin{array}{l}\text { Taux } \\
\text { vacances } \\
\text { médecins } \\
\text { spécialistes }\end{array}$ & $\begin{array}{l}\text { Taux de vacances } \\
\text { infirmières/sages- } \\
\text { femmes }\end{array}$ & $\begin{array}{l}\text { Part des frais de } \\
\text { personnel } \\
\text { contractuel sur total } \\
\text { frais personnel }\end{array}$ \\
\hline $\begin{array}{l}\text { Ayrshire } \\
\text { Arran }\end{array}$ & 5,01 & 13,9 & 0,4 & 2,7 \\
\hline Borders & 4,36 & 7,2 & 3,7 & 3,3 \\
\hline $\begin{array}{l}\text { Dumfries } \\
\text { Galloway }\end{array}$ & 5,08 & 14,5 & 4,4 & 6,2 \\
\hline Fife & 5,12 & 12,6 & 2,4 & 2,9 \\
\hline Forth Valley & 5,1 & 3,6 & 3,4 & 2,4 \\
\hline Grampian & 4,62 & 6,2 & 7,3 & 3,4 \\
\hline
\end{tabular}




\begin{tabular}{|l|l|l|l|l|}
\hline $\begin{array}{l}\text { Greater } \\
\text { Glasgow } \\
\text { Clyde }\end{array}$ & 5,39 & 3,9 & 4,3 & 2 \\
\hline Highland & 5,09 & 6,1 & 5,4 & 4,3 \\
\hline Lanarkshire & 5,2 & 10,1 & 3,3 & 3,3 \\
\hline Lothian & 5,02 & 3,5 & 1,6 & 3 \\
\hline Orcades & 5,1 & 37 & 8,8 & 5,2 \\
\hline Shetland & 5,2 & 0 & 8,1 & 6,8 \\
\hline Tayside & 5,04 & 6,9 & 2,9 & 1,9 \\
\hline Hébrides \\
extérieures
\end{tabular}

Source : AUDITOR GENERAL, NHS in Scotland 2016, Édimbourg, 2016, pp. 30-31.

Tableau 5 : Résultats des objectifs de prise en charge dans les health boards en 2017 : parcours de soins

\begin{tabular}{|c|c|c|c|c|}
\hline $\begin{array}{l}\text { Conseil et } \\
\text { objectif }\end{array}$ & $\begin{array}{lll}\text { Du renvoi } & \text { au } \\
\text { spécialiste } & \text { vers } & \text { le } \\
\text { traitement } & \text { en } & 18 \\
\text { semaines }(90 \%) & \end{array}$ & $\begin{array}{l}\text { Renvoi au } \\
\text { spécialiste en } 12 \\
\text { semaines (95\%) }\end{array}$ & $\begin{array}{l}\text { Traitement hospitalier } \\
\text { débutant en } 12 \\
\text { semaines suivant } \\
\text { décision }(100 \%)\end{array}$ & $\begin{array}{l}\text { Urgences, } \\
\text { prise } \\
\text { charge en } \\
(95 \%)\end{array}$ \\
\hline $\begin{array}{l}\text { Ayrshire \& } \\
\text { Arran }\end{array}$ & 73,6 & 82,6 & 86,6 & 93,7 \\
\hline Borders & 90 & 90,8 & 95,7 & 93,2 \\
\hline $\begin{array}{l}\text { Dumfries \& } \\
\text { Galloway }\end{array}$ & 89,5 & 92 & 86,3 & 93,7 \\
\hline Fife & 89,1 & 95,5 & 91,2 & 95,2 \\
\hline Forth valley & 79,4 & 91,6 & 63,5 & 97,2 \\
\hline Grampian & 74,5 & 72,6 & 74,4 & 96,1 \\
\hline $\begin{array}{l}\text { Greater } \\
\text { Glasgow \& } \\
\text { Clyde }\end{array}$ & 89,7 & 86 & 87,2 & 90,7 \\
\hline Highland & 78,2 & 63,4 & 75,8 & 96,8 \\
\hline
\end{tabular}




\begin{tabular}{|l|l|l|l|l|}
\hline Lanarkshire & 78,7 & 83,4 & 66,7 & 90 \\
\hline Lothian & 79,1 & 72,7 & 81,4 & 95,7 \\
\hline Orcades & 94,3 & 67,8 & 90,3 & 97,5 \\
\hline Shetland & 84,2 & 68,1 & 98,1 & 97,1 \\
\hline Tayside & 86,7 & 86 & 81,2 & 98,6 \\
\hline $\begin{array}{l}\text { Hébrides } \\
\text { extérieures }\end{array}$ & 95,6 & 95,6 & 100 & 99,3 \\
\hline Écosse & 83,2 & 80,7 & 82,2 & 93,8 \\
\hline
\end{tabular}

Source : AUDITOR GENERAL, NHS in Scotland 2017, Édimbourg, 2017, annexe 3.

Tableau 6: Résultats des objectifs de prise en charge dans les health boards en 2017 : pathologies spécifiques

\begin{tabular}{|c|c|c|c|c|}
\hline $\begin{array}{l}\text { Conseil et } \\
\text { objectifs }\end{array}$ & $\begin{array}{l}\text { Problèmes } \\
\text { mentaux de } \\
\text { mineurs, rendez- } \\
\text { vous en } 18 \\
\text { semaines }(90 \%)\end{array}$ & $\begin{array}{l}\text { Problèmes drogue } \\
\text { ou alcool, rendez- } \\
\text { vous en } 3 \\
\text { semaines }(90 \%)\end{array}$ & $\begin{array}{l}\text { Cancer: } 62 \text { jours } \\
\text { entre renvoi au } \\
\text { spécialiste et } \\
\text { traitement (95\%) }\end{array}$ & $\begin{array}{l}\text { Cancer: traitement } \\
\text { débutant dans les } \\
31 \text { jours suivant la } \\
\text { décision }(95 \%)\end{array}$ \\
\hline $\begin{array}{l}\text { Ayrshire \& } \\
\text { Arran }\end{array}$ & 93,8 & 96,8 & 92,8 & 99,7 \\
\hline Borders & 98,4 & 94,4 & 95,1 & 98,3 \\
\hline $\begin{array}{l}\text { Dumfries \& } \\
\text { Galloway }\end{array}$ & 100 & 97,1 & 96,3 & 96,5 \\
\hline Fife & 84,5 & 96,6 & 80,5 & 97,8 \\
\hline Forth valley & 99,7 & 98,7 & 89,3 & 96,6 \\
\hline Grampian & 45,2 & 93,3 & 86,2 & 92,2 \\
\hline $\begin{array}{l}\text { Greater } \\
\text { Glasgow \& } \\
\text { Clyde }\end{array}$ & 98 & 96,8 & 83,3 & 93,9 \\
\hline Highland & 96 & 84 & 87,2 & 97,8 \\
\hline Lanarkshire & 87,2 & 99,8 & 95,9 & 96,9 \\
\hline Lothian & 47,8 & 83,3 & 90,6 & 93,6 \\
\hline Orcades & 100 & 100 & 84,6 & 100 \\
\hline
\end{tabular}




\begin{tabular}{|l|l|l|l|l|}
\hline Shetland & 100 & 88,9 & 94,1 & 100 \\
\hline Tayside & 95,2 & 96,7 & 89,6 & 93,1 \\
\hline $\begin{array}{l}\text { Hébrides } \\
\text { extérieures }\end{array}$ & 100 & 94,2 & 85 & 100 \\
\hline Écosse & 83,6 & 94,9 & 88,1 & 94,9 \\
\hline
\end{tabular}

Source : AUDITOR GENERAL, NHS in Scotland 2017, Édimbourg, 2017, annexe 3.

\section{NOTES}

1. Des structures publiques, et peu coûteuses pour les patients, avaient été instaurées dans des régions pauvres, les Highlands en 1913, les environs de Glasgow en 1941 (les hôpitaux militaires étant ouverts aux civils). SP, OR, 26 juin 2018, col. 71.

2. HAMILTON David, "Health", in MACDONALD Margo (dir), The Radical Approach, Édimbourg, Palingenesis Press, 1976, p. 74.

3. LUdBrook Anne, theodossiou Ioannis, GEROVA Vania, «Health and Deprivation " in ERMISCH John, WRIGHT Robert (dir), Changing Scotland, Bristol, Policy Press, 2005, p. 99. Le qualificatif avait d'abord été attribué à l'économie britannique.

4. DAYAN Mark, EDWARDS Nigel, Learning from Scotland's NHS, Londres, Nuffield Trust, juillet 2017.

5. SCOTTISH CONSTitutional CONVEntion, Scotland's Parliament, Scotland's Right, Édimbourg, 1995.

6. AG, NHS in Scotland, Édimbourg, Audit Scotland, 2017, 10.

7. BEVAN Gwyn, KARANiKolos Marina, EXLEY Jo, NOLTE Eileen, ConNolly Sheelah, MAYs Nicholas, The Four Health Systems of the United Kingdom, Londres, Nuffield Trust, 2014, p. 49.

8. SP, OR, 2 mai 2018, col. 40 (Shona Robison, ministre de la Santé).

9. Il a supprimé le conseil d'Argyll \& Clyde, lourdement déficitaire en 2005 et évincé la direction de Tayside qui avait puisé dans un fonds protégé alimenté par des dons (2018). Il existe aussi 7 health boards nationaux, axés sur la santé publique, la qualité des soins, la formation du personnel, les ambulances, les urgences psychiatriques, les appels téléphoniques, les listes d'attentes.

10. SP, OR, 4 octobre 2018, col. 49 (Jeane Freeman, ministre de la Santé).

11. HUNTER D., « The Reorganised Health Service ", Scottish Government Yearbook, 1977, p. 30-32.

12. MCTAVISH Duncan, "Scottish and English Health Policy from 1948 to the 1973 Reforms ", Scottish Affairs, $n^{\circ}$ 51, 2005, p. 60. BRUCE Allan, FORBES Tom, « From Competition to Collaboration in the Delivery of Health Care», Scottish Affairs, $n^{\circ} 34,2001$, p. 109. woods Kevin, "Scotland's Changing Health System ", in WOODS Kevin, CARTER David (dir), Scotland's Health and Health Services, Londres, Stationery Office, 2003, chapitre 1.

13. GREER Scott, Territorial Politics and Health Policy, Manchester, Manchester University Press, 2004, p. 79.

14. HEENAN Deirdre, BIRRELl Derek, The Integration of Health and Social Care in the UK, Londres, Palgrave Macmillan, 2018, p. 68.

15. MILNE Robin, "Competitive Tendering of NHS Hotel Services in Scotland », Scottish Affairs, $n^{\circ} 3$, 1993, p. 145. Les directives publiées en 1983 ont privilégié la restauration, déconseillant la blanchisserie en raison de la faiblesse du marché écossais.

16. SP, OR, $1^{\mathrm{er}}$ novembre 2017, col. 53 (Maree Todd). 
17. Le gouvernement britannique n'avait pas exigé d'exceptions dans les traités négociés par l'UE (accord sur le commerce des services dans le cadre de l'OMC en 2005, projet de traité transatlantique). DODDS Anneliese, «The Politicisation of Trade in Health and Education Services", Scottish Affairs, n46, 2014, p. 56-75 ; SP, OR, 19 août 2014, col. 33578, Alex Neil, ministre de la Santé; SP, HSC, OR, 6 mars 2018, p. 11 ; HC, Hansard, 23 mai 2018, Chris Stephens (SNP).

18. SP, Culture, Tourism, Europe and External Relations Committee, Brexit-What Scotland Thinks, Édimbourg, SP 64, 2017, p. 80.

19. SG, Brexit, Protecting What Matters, Édimbourg, 2018, p. 14, 18.

20. Les personnes indisponibles étaient soustraites des listes (jusqu'en 2008), tandis que le nombre de patients sans garantie était en progression. AG, Tackling Waiting Times in the NHS in Scotland, Édimbourg, Audit Scotland, 2006.

21. SP, OR, 24 février 2011, col. 33553.

22. AG, 2016, op. cit., p. 20.

23. SP, OR, 24 octobre 2018, col. 2.

24. SP, OR, 12 mars 2009, col. 15811 (Ian McKee, SNP), 15816 (Richard Simpson, travailliste).

25. GREER Scott, WILSON Iain, STEWART Ellen, DONNELLY Peter, Health Board Elections and Alternative Pilots, Édimbourg, SG, 2012, 3.9.

26. Sondages YouGov (octobre 2015-avril 2017), IPSOS-MORI (mai 2017), www.whatscotlandthinks.org, consulté le $1^{\mathrm{er}}$ juin 2018.

27. oRMSTON Rachel, curTice John, «Attitudes Towards a 'British Institution' », Scottish Affairs, $\mathrm{n}^{\circ}$ 61, 2007, p. 69. KEATING Michael, LINEIRA Robert, « Getting to a Wealthier and Fairer Scotland », in KEATING Michael (dir), A Wealthier, Fairer Scotland, Édimbourg, Edinburgh University Press, 2017, p. 134-135.

28. SP, OR, 25 janvier 2006 (Andy Kerr, ministre de la Santé).

29. SP, OR, 18 novembre 2010, col. 30581.

30. SP, OR, 5 décembre 2007, col. 4065 (Des McNulty).

31. MCARDLE Helen, «Free Prescription 'Save NHS Money' Despite Soaring Bill for Ageing and Obesity », The Herald, 5 octobre 2017, p. 6.

32. SP, OR, 18 novembre 2004, col. 12116.

33. SP, OR, 20 juin 2002, col. 13976 (Tommy Sheridan).

34. SP, OR, 19 février 2014, col. 27917 (Aileen Campbell, ministre des Enfants).

35. MCADAMS Rachel, Evaluating Universal Infant Free School Meals, Scotland, Édimbourg, NHS Health Scotland, 2016, p. 7.

36. вRоокs Libby, «Council Plans Free School Meals All Year to Tackle 'Holiday Hunger' », The Guardian, 16 février 2018.

37. SP, OR, 25 avril 2018, col. 117.

38. SP, OR, 18 novembre 2014, Kenneth MacAskill, ministre de la Justice, aurait souhaité la dévolution des pouvoirs de sanctions.

39. SP, OR, 16 novembre 2005, George Lyon, ministre délégué aux Finances.

40. SP, OR, 10 novembre 2011, col. 30243.

41. SP, OR, 24 mai 2012, col. 9426 (Jackie Baillie), 9427 (Jackson Carlaw).

42. SUPREME COURT, Scotch Whisky Association and others v. Lord Advocate and Another, Londres, 2017, paragraphe 63.

43. The Herald, $1^{\mathrm{er}}$ mai 2018 , p. 1. Le prix minimum d'une bouteille est porté à 14 livres pour le whisky, 4,88 livres pour le vin.

44. SP, OR, 21 novembre 2017, col. 19.

45. SP, OR, 30 juin 2005, col. 18709 (Kerr).

46. SP, OR, 27 janvier 2010, col. 23160 (Sturgeon).

47. SP, HSC, OR, 20 mars 2018, col. 30. 
48. PATERSON Lindsay, BECHHOFER Frank, MCCRONE David, Living in Scotland, Édimbourg, Edinburgh University Press, 2004, p. 157.

49. BROOKS Richard, "Scotland's Health" in INGRAM Keith, LOVE James (dir), Understanding the Scottish Economy, Oxford, Martin Robertson, 1983, p. 107.

50. MCCRONE David, The New Sociology of Scotland, Londres, Sage, 2017, p. 115.

51. PATERSON et al., op.cit., p. 200.

52. MCCRONE, op.cit., p. 122-123.

53. HAMILTON David, "Health and Health Care in West Central Scotland ", Scottish Government Yearbook 1978, p. 79.

54. CHURCH Jenny (dir), Regional Trends, Londres, HMSO, 1994, p. 102. MCLEAN Joanne, CHRISTIE Joanne, GRAY Lindsay, Scottish Health Survey, Édimbourg, SG, 2016, p. 105.

55. PATERSON et al., op.cit., p. 136. MCKENDRICK John, « What is Life Like for People Experiencing Poverty?", in MCKENDRICK John, MOONEY Gerry, sCOTT Gill, DICKIE John, MCHARDY Fiona (dir), Poverty in Scotland 2016, Londres, CPAG, 2016, p. 120-121.

56. SP, HSC, Stage 1 Report on the Alcohol (Minimum Pricing) (Scotland) Bill, Édimbourg, 2012, paragraphe 10.

57. PATERSON et al, op.cit., p. 133-134.

58. Robertson Tony, marsden Sara, Kapilashrami Anuj, «A Public Health Politics that is People's Health », in HASSAN Gerry, BARROW Simon (dir), A Nation Changed ?, Édimbourg, Luath Press, 2017, p. 127.

59. AG, NHS in Scotland, Édimbourg, 2016, p. 24.

60. HAMILTON, 1978, op. cit., p. 82. AG, Health Inequalities in Scotland, 2012, p. 11.

61. HC, Scottish Affairs Committee, Demography of Scotland and the Implications for Devolution, Londres, 2016, p. 22.

62. SG, Scotland's Place in Europe, Édimbourg, 2018, 130.

\section{RÉSUMÉS}

Depuis 1999, le Parlement et le gouvernement écossais se sont efforcés d'améliorer la santé de la population écossaise, en restaurant un service public fondé sur la coopération et non sur la concurrence, tout en concevant des politiques publiques visant à modifier les comportements individuels. En dépit des succès obtenus, ils n'ont pu éviter la politisation de cet enjeu, dans un contexte de restrictions budgétaires croissantes et d'incertitudes institutionnelles.

Since 1999, Scotland's Parliament and government have striven to improve the health of the Scottish people by restoring a public service based on co-operation and not on competition, while devising policies aimed at modifying the behaviour of individuals. Even though they have achieved some success, they have proved unable to avoid politicizing the issue. Tensions have been fuelled by tighter budgetary constraints and constitutional upheavals. 
INDEX

Mots-clés : dévolution, santé, SNP, enfants, inégalités

Keywords : devolution, health, SNP, children, inequality

\section{AUTEUR}

\section{EDWIGE CAMP-PIETRAIN}

Université Polytechnique des Hauts-de-France (Valenciennes) 